\title{
LA-UR-09- 07307
}

Approved for public release; distribution is unlimited.

Title: DEFECT DETECTION IN CONCRETE MEMBERS

Author(s): H. Sezer Atamturktur, Civil Engineering Dpt., Clemson University

Intended for:

$28^{\text {th }}$ SEM International Modal Analysis Conference (IMAC-XXVIII), Jacksonville, Florida, February 1-4, 2010

Los Alamos National Laboratory, an affirmative action/equal opportunity employer, is operated by the Los Alamos National Security, LLC for the National Nuclear Security Administration of the U.S. Department of Energy under contract DE-AC52-06NA25396. By acceptance of this article, the publisher recognizes that the U.S. Government retains a nonexclusive, royalty-free license to publish or reproduce the published form of this contribution, or to allow others to do so, for U.S. Government purposes. Los Alamos National Laboratory requests that the publisher identify this article as work performed under the auspices of the U.S. Department of Energy. Los Alamos National Laboratory strongly supports academic freedom and a researcher's right to publish; as an institution, however, the Laboratory does not endorse the viewpoint of a publication or guarantee its technical correctness.

Form $836(7 / 06)$ 
This page is left blank intentionally. 


\title{
Defect Detection in Concrete Members
}

\author{
Sezer Atamturktur
}

A rock pocket is a deficient volume within hardened concrete consisting of coarse aggregate and voids that reduce the overall stiffness of the concrete members. The leakage of wet concrete from the form, segregation, or insufficient consolidation during concrete placement may leave rock pockets in concrete construction. This study is concerned with the detection of internal defects, particularly rock pockets, in reinforced concrete beam members. Impact hammer tests are completed on two concrete beams of same properties but defected with rock pockets. The finite element models of these beams are segmented, and the stiffness properties of these segments are independently calibrated using Bayesian calibration techniques. Through model calibration, not only the locations but also the severity of rock pockets is detected.

\section{Introduction:}

A rock pocket is a local, internal defect, which may be left in concrete members when the mortar paste fails to fill the space around the coarse aggregates. The presence of these internal defects typically reduces the load capacity of a concrete member, the severity of this reduction being proportional to the dimensions of the defect. Depending on the size and location of internal defects as well as construction factors (i.e., water-to-cementitious materials ratio, curing, environmental conditions, etc.), degrading effects of these internal defects may either be evident immediately or present themselves after the structural element is in service. For instance, in prestressed precast beams, such internal defects, if extensive or if located near the anchorage can cause compression failure during the stressing of tendons. Also, the presence of such inherent construction defects tends to accelerate the degrading effects of aging, operational and environmental conditions.

Rock pockets, being internal defects, cannot be located through visual inspection, thus traditional techniques of detecting these defects required drilling samples. The traditional techniques were labor intensive, time consuming and semi-invasive. Because the civil engineering community has a pervasive interest in the ability to detect defects in concrete members, a number of nondestructive evaluation techniques have been developed. These techniques are primarily based on the mechanical principle of stress wave propagation. Examples of such methods include acoustic impact method (sounding), ultrasonic pulse-velocity method, ultrasonic pulse-echo method, and impact-echo method. Although these localized nondestructive defect detection methods exhibited a level of success in detecting damage near the surface of the member where measurements are taken, they have a drawback of requiring a priori knowledge about the vicinity of the defect as well as access to the damaged region to perform nondestructive testing. In typical applications, the presence of internal defects, as well as their location, severity and kind are not known a priori, any defect detection method must be capable of detecting defects without prior knowledge of these aspects. The goal of this study is to make a small step towards the development of a rapid assessment tool to detect not only the presence but also the location 
and severity of internal defects. The proposed method also shows some success in distinguishing between honeycombing and rock pockets in reinforced concrete beams.

An important step in decision making regarding the maintenance of a concrete element is the classification of the defect- that is the determination of type and severity of the defect. Ultimately, one needs to determine whether the degrading effects of a defect are significant to mandate repair schemes. Therefore, any defect-detection method must be able to determine the type and quantify the severity of defect to be of any use. Moreover, knowing the precise location of the defect would make elaborate and invasive repair schemes possible, which typically include removal of concrete and mortar injections. The proposed method has the potential to provide, in real time, reliable information regarding the defects in the concrete member. Therefore, one output of the proposed approach may be the rapid and inexpensive assessment of the conditions of existing concrete structures. Moreover, the ability to determine the presence of defects, or lack thereof, when made readily available, can also provide venues for quality assessment of prestressed, precast concrete members.

\section{Background in Vibration Based Defect \& Damage Detection:}

The problem of defect detection is not any different than the widely studied problem of damage detection. The assessment of defects in a system can either be calculation based or experimentation based. However, neither of these approaches can yield a complete set of information about internal defects. By complete set of information, we mean the steps developed by Rytter (1993) for damage detection: detection, location, quantification and prognosis. These steps can easily be transferred to the problem of defect detection. For instance, the detection problem can be handled by using pattern recognition or novelty detection approaches using solely measurements without a strict need for a simulation model (Worden 1997, Worden 2000). However for localization and quantification, almost certainly one must combine a simulation model with measurements. Therefore, the current trend is integrating the experimental measurements with FE model calculations in order to obtain a calibrated FE model which can be used to make predictions about the state of the structural element which cannot be observed through measurement due to a variety of reasons. Detection of damage in structural members through model calibration against vibration measurements has received significant attention over the last two decades. A detailed overview of the pertinent literature is deemed out of the scope of this paper, but interested readers are invited to look at Doebling et al. (1998) and Sohn et al. (2004).

Theoretically, if all the modes of a structure were identified experimentally, the optimizationbased calibration would be able to calibrate all parameters necessary to construct a FE model. In other words, one can effectively calibrate the individual properties of each finite element. Such an approach would also enable the representation of local variability of material properties as well as localized cracks, an important uncertainty source for civil engineering structures. Aoki et al. (2005) and in a refined version Aoki et al. (2008) presented the results of such study. A dynamic identification, FE modeling and FE model calibration campaign was applied to a brick chimney. The FE model of the chimney was built with 20 -node isotropic solid elements and a correction factor was assigned for both the mass and stiffness of each finite element. Using a 
sensitivity based calibration approach, the elemental mass and stiffness correction factors were sought. To alleviate the inevitable incompleteness of the measurements, a weighting function was applied to eliminate the finite elements with low sensitivity. The element stiffness at the base of the chimney was observed to be reduced, while at the corners, the stiffness was observed to be increased. The author explained the former by chimney soil interaction and the latter by the iron angles at the corners. The way calibration is handled in this study can be considered as a reconstruction-based approach. Such an approach has been demonstrated to be successful in a number of studies when the number of finite elements is low and when the available measurements are in high quality and quantity. However, for more complex problems with larger numbers of finite elements, this approach renders the damage detection an undetermined problem.

However, Vestroni (2008) emphasizes that a damage detection problem can also be represented as a determined problem. Formulating damage in terms of location and severity of damage instead of the reconstruction of the entirety of the member elemental matrices eliminates the need for excessively large amounts of experimental information. Therefore, the mass and stiffness parameters of a structure can be considered to be uniform and equal to the baseline value throughout the structure except at some distinct locations where damage (or defect) is present. Such an approach can effectively be applied to the present problem while aiming at detecting defects in concrete structures. The construction defects considered in this study are localized and typically few in number, therefore only a limited number of parameters would be sufficient to describe the defects. However, the absence of knowledge about their location, type and severity makes the evaluation of a large number of candidate scenarios necessary. How this evaluation is completed will be discussed in the following section.

\section{Model Calibration Procedure:}

In this study, the FE model parameters that define the internal defects will be calibrated using experimental measurements obtained from the concrete beam members cast with specific defects. The details of the calibration methodology adapted herein, which can be found in Higdon et al. (2007-2008) and Kennedy and O'Hagan (2000), will be overviewed in the following paragraphs.

The calibration methodology adapted herein treats both the FE model parameters and the FE model solution probabilistically. When both the FE model input and FE model output are treated in a probabilistic manner, two types of uncertainty propagation are required. Determining how much uncertainty in the selected calibration parameters causes variability in the output is referred to as forward uncertainty propagation. The inverse uncertainty propagation, in contrast, investigates the sources of uncertainty in the output by focusing on the variability of calibration parameters [Figure 1].

The forward propagation of uncertainty consists of a family of computer runs repeated at the sampled input parameters to observe the variability in the FE model outcomes. As long as a large enough number of samples are generated, this sampling approach converges to the actual distribution of the output parameter. The number of necessary samples depends strictly on the 
order of complexity of the sampled behavior and on the type of sampling design. Inverse propagation of uncertainty is computationally more involved as it conceptually requires the FE model to be inverted. However, for real engineering solutions, the requirement of inverting an FE model is practically prohibitive because of the discretization based approximate approach inherent in the FE analysis.

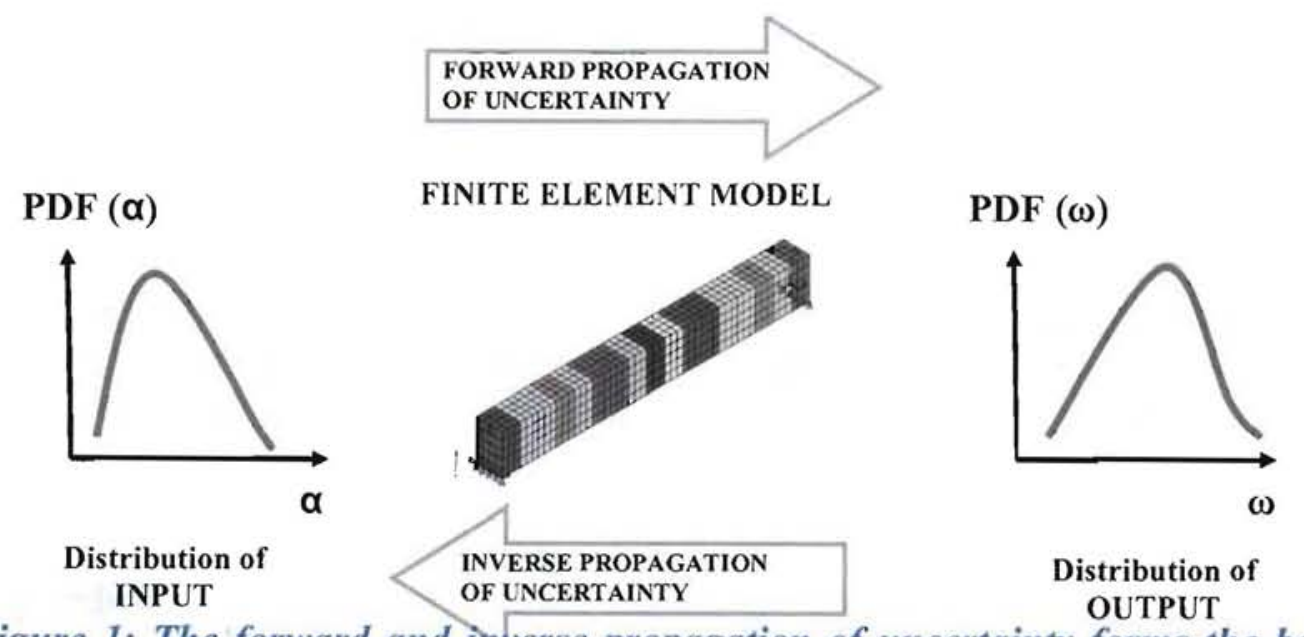

Figure 1: The forward and inverse propagation of uncertainty forms the basis of stochastic calibration.

In stochastic model calibration, the inverse propagation of uncertainty forms the basis of the statistical inference problem. In theory, if the uncertainty in input can be reduced, the uncertainty in the output will also be reduced. Thus, the inverse propagation of uncertainty is usually called upon in model calibration activities to seek the sources of the output uncertainty.

In the formulation adapted in this study, the inverse propagation of uncertainty is replaced by a large cohort of forward propagation of uncertainty via Markov Chain Monte Carlo (MCMC) sampling. MCMC performs a random walk in the domain defined by the calibration parameters defined by $\alpha 1$ and $\alpha 2$ in Figure 2 according to the probability distribution of the calibration parameters: $\mathrm{P}(\alpha 1)$ and $\mathrm{P}(\alpha 2)$. In the absence of better knowledge, the probability distribution of calibration parameters can be assigned equal probability for all possible values between an upper and lower limit. During each random walk, the model calculates the selected output response according to the sampled parameter values. Comparative feature is defined by $\omega$ in Figure 2 . The acceptance criterion for the sampled parameters is guided by the likelihood estimation $\mathrm{P}(\omega \mid \alpha 1$, $\alpha 2$ ). The current sample point (in the domain of the calibration parameters $\alpha 1$ and $\alpha 2$ ) is rejected if it reduces the likelihood that the set of calibration parameters is correct. If the current sample point is rejected, the random walk returns the last accepted point and the probability distributions of the calibration parameter remains unchanged. However, if the sample point is accepted, the posterior distributions of calibration parameters are obtained. These posterior distributions of the calibration parameters become the prior distributions in the next random walk. According to the current priors, MCMC performs another random walk from the last accepted point to the next point [Figure 2]. 
The repeated feedback in Bayesian inference progressively characterizes not only the posterior distributions of the calibration parameters, defined by $\alpha 1$ and $\alpha 2$ in Figure 2, but also the hyperparameters of the Gaussian Process Models (GPM), a fast running surrogate used to replace the computationally expensive FE simulations. If there are ' $n$ ' MCMC iterations, there will be ' $n$ ' different sets of estimates for hyperparameters and calibration parameters that are accepted by the likelihood function. By using the hyperparameters of the GPM of the discrepancy term, one can now construct the error model, and by using the calibration parameter values along with the hyperparameters of the simulation emulator, one can construct the surrogate model. Thus, the method defines all possible values of $\eta(x ; t)$ and $\delta(x)$ that, when added together, reproduce the experiments.

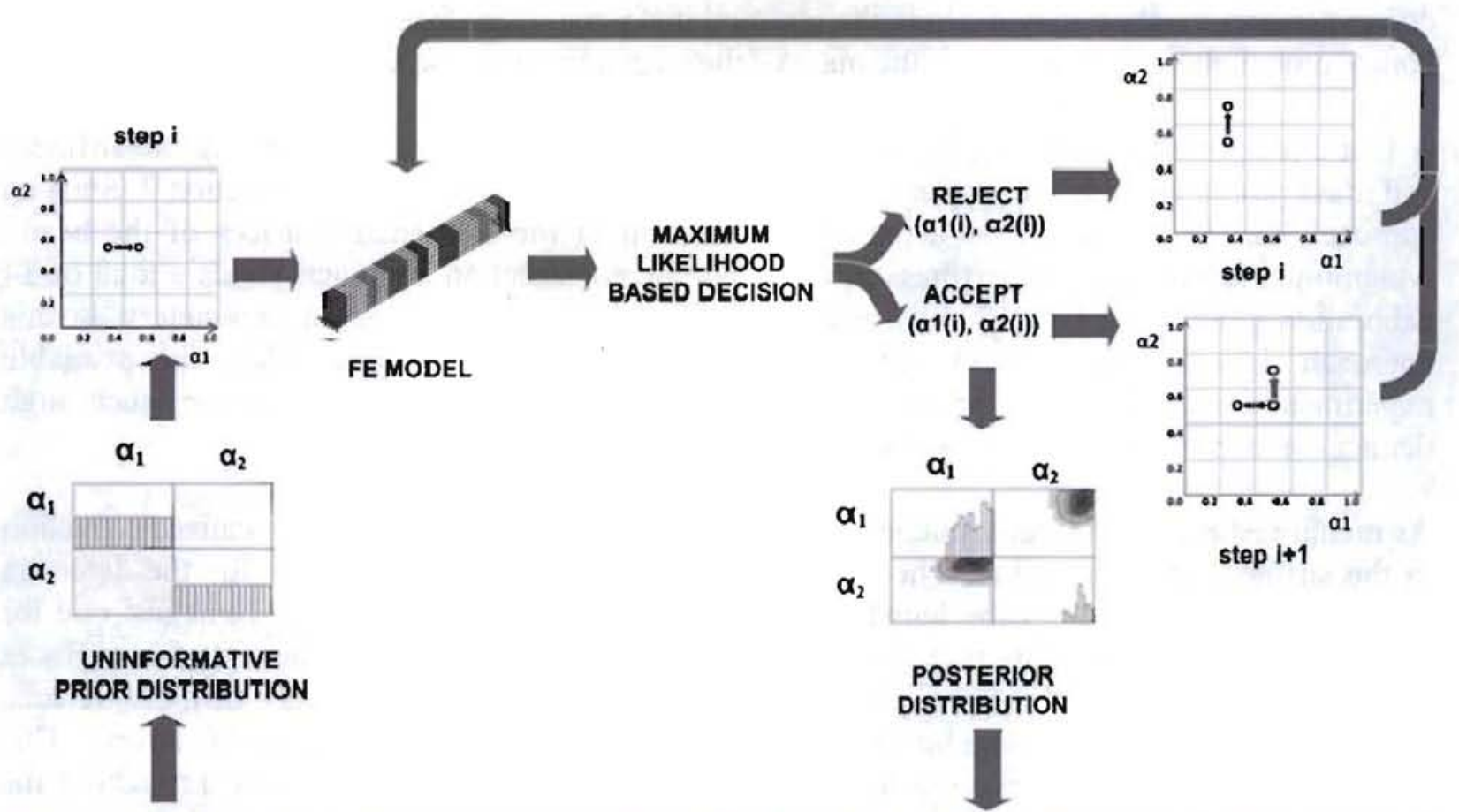

Figure ENTER a adapted procedure combines Markov chain IEXTTe Carlo with the context of Bayesian inference.

Experiments, are subject to random and bias errors, however they constitute our best representation of physical reality. Thus, the calibration process is obligated to tolerate experimental errors. Experimental uncertainty is typically categorized in two groups: (1) measurement uncertainty, for instance due to instrumentation and data processing, and (2) natural variability of the structure, for instance in heterogeneous materials. Experimental errors are commonly incorporated in the analysis with the help of probability theory. Typically, the error term is defined as a zero-mean Gaussian random variable. Such an approach is best justified by the central limit theorem. If we assume that the experiments are immune from systematic errors, the experimental error can be considered to be a summation of a large number of independent processes. According to the central limit theorem, these sources would collectively converge to a normal distribution (Hogg and Craig 1978). 
The mean estimates of the posterior distribution provide the most likely values for the calibration parameters. These values, when obtained through stochastic calibration, can later be used in a deterministic study. The standard deviation of the posterior distributions captures the remaining uncertainty in the parameter values.

\section{Proof-Concept}

A key aspect of calibration based defect detection is the suitable parameterization of the defect of interest. For instance, rock pocket defects result in localized reduction in stiffness while the change in mass, relatively speaking, is negligible. However, honeycomb defects result in reduction in both stiffness and mass. If the defect is strictly known to be a rock pocket, then the defect parameterization may exclude the mass-related parameters from calibration. However, for honeycomb defects to be detected, the mass of the structure must also be parameterized.

A first attempt for approaching the defect detection problem is through calibrating the stiffness and mass properties of all seventeen units individually, as discussed earlier in Section 2. Such an approach can be considered as a total reconstruction of the elemental matrices of the beam. Assuming a lump mass and stiffness model, a total reconstruction approach yields a total of 34 calibration parameters $(\mathrm{i}=1: 17 ; \mathrm{k}(\mathrm{i})$ and $\mathrm{m}(\mathrm{i}))$. The number of calibration parameters, in this approach, is quite high, which would result in non-unique solutions unless the available experimental information is in sufficiently high quantity and quality. However, such high demand on experimentation is commonly not feasible.

As mentioned earlier, the consequence of rock pocket defects is mainly some localized reduction in the stiffness of the member. Therefore, an alternative formulation is to define the defect in terms of its ordinate across the length of the beam $(x)$ and two reduction percentages: one for stiffness $(y)$ and one for mass $(z)$ [Figure 3]. In the presence of one defect, this approach reduces the model calibration to three parameters (x, y and $\mathrm{z}$ ) only. However, in case of more defects, the number of calibration parameters increases proportionally to the number of defects. This approach accepts the stiffness and mass properties of the beam to be identical throughout the member but at certain location where the damage is present.

The consequence of internal defects can be represented in different precision levels varying from a very refined finite element or a substructure consisting of several finite elements. The desired precision will be reflected in the parameterization of the defect, and naturally a larger amount of experimental information will be necessary for improved precisions. In this study, because only lower order vibration modes were available, only a relatively crude defect model can be identified.

Defining the defect in terms of mass and stiffness reduction factors as well as a location indicator reduces the number of calibration parameters significantly. We believe that a more sophisticated and precise approach than the one adapted herein is unlikely to lead to practical tools for rapid assessment of concrete structures. This approach however, has the drawback that a good estimate of the number of defects should be available a priori. This drawback is more pronounced especially when only the changes to natural frequencies are measured as defect indicating 
symptoms. For instance, the scenario \#1 given below illustrates the problem of uniqueness of calibration.

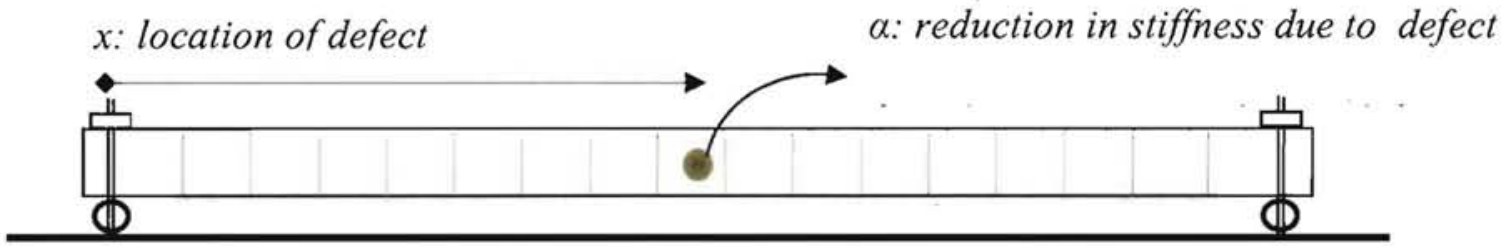

Figure 3: Defect is defined with a location parameter and a reduction parameter.

A FE model of a simply supported beam is developed in ANSYS. The beam has a cross-section of $15 \mathrm{~cm} \times 22.5 \mathrm{~cm}$ and a length of $1.95 \mathrm{~m}$. The beam is assumed to be simply supported at both ends. A uniform $30 \mathrm{GPa}$ Young's Modulus and $2400 \mathrm{~kg} / \mathrm{m} 3$ density are assumed. The beam model has a total of 17 sections, each of which are assigned individual mass (density) and stiffness (Young's modulus) properties [Figure 3]. The mesh of the beam FE model is given in Figure 4. A more refined mesh was deemed unnecessary because an element with a quadratic shape function was used. For defect indicators, the first bending modes in the Y direction are considered [Figure 5].
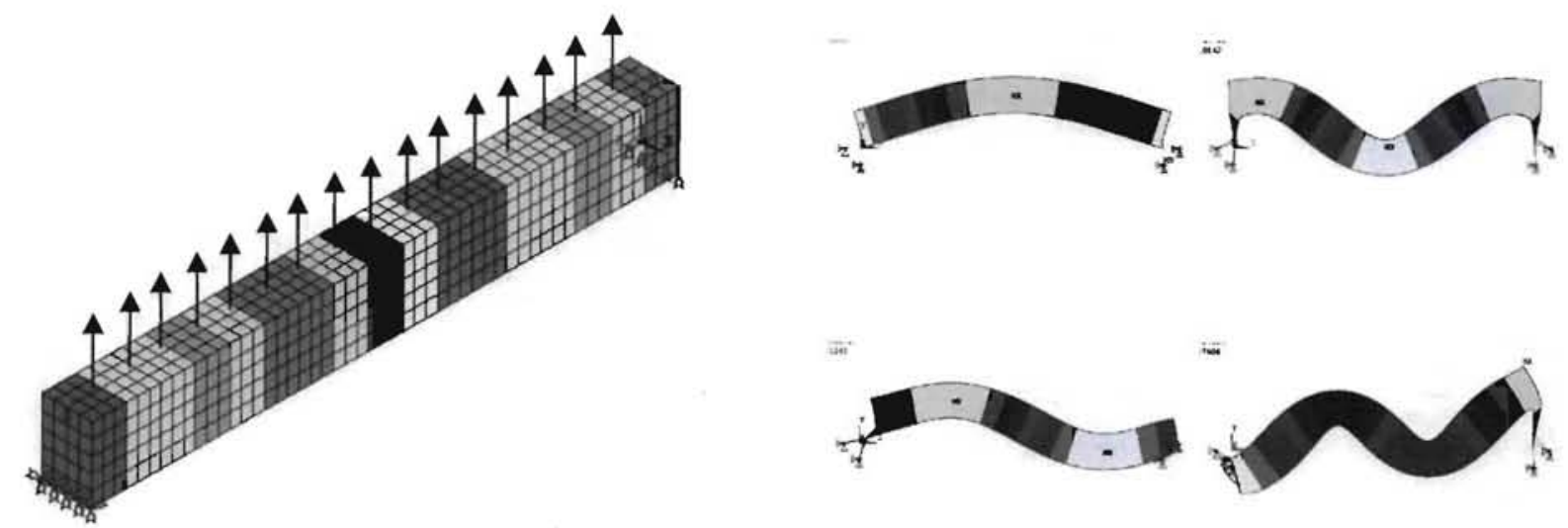

Figure 4: Finite element model of a simply supported beam.

Figure 5: The first five bending modes of the beam are used for defect detection.

- Scenario \#1: Detecting single rock-pocket defect using only natural frequencies.

We begin by assuming a single defect close to the mid-length of the beam [Figure 6]. We will assume to know the exact number and type of the defect: one rock-pocket defect. Therefore, the calibration parameters will be reduced to two parameters only: $x$ and $\alpha$. This scenario represents the ideal conditions for defect detection approach proposed herein. 
Virtual experimental data of the natural frequencies of the first four bending modes are simulated by the FE model with the prescribed defect $(x=7$ and $\alpha=0.75)$. Virtual experiments are deterministic in nature, however to account for potential variability during physical experiments, a 2.5 percent experimental uncertainty is assumed. The goal is to retrieve the 'correct' $\mathrm{x}$ and $\alpha$ value used to generate the virtual experiments.

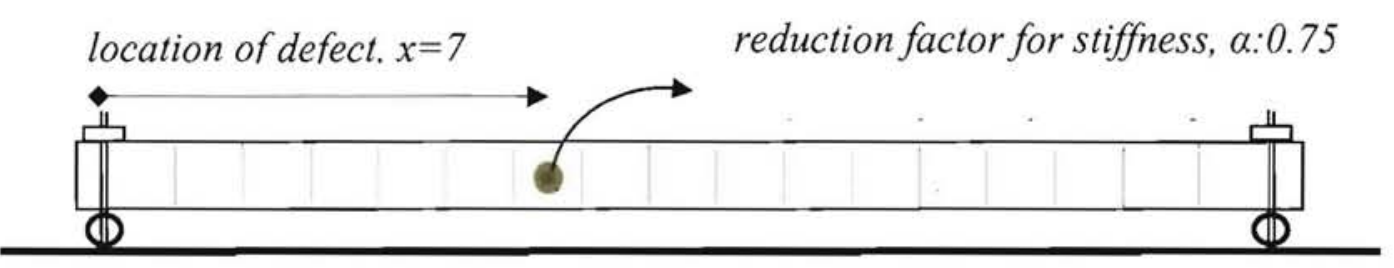

Figure 6: Characteristics of the virtual defect simulated using the finite element model.

In this framework, the maximum allowable changes for the calibration parameters are assigned through the domain within which the MCMC algorithm performs random walks. The defect can be located in any of the 17 bins of the beam FE model. Therefore, the $x$ parameter is allowed to be $(x=1: 17)$. The defect is expected to reduce the Young's Modulus of concrete, therefore a range from $21 \mathrm{GPa}$ to $30 \mathrm{GPa}$ is considered for the stiffness reduction parameters $(\alpha=0.7: 1)$. Five different levels of stiffness reduction are considered between these lower and upper bounds.
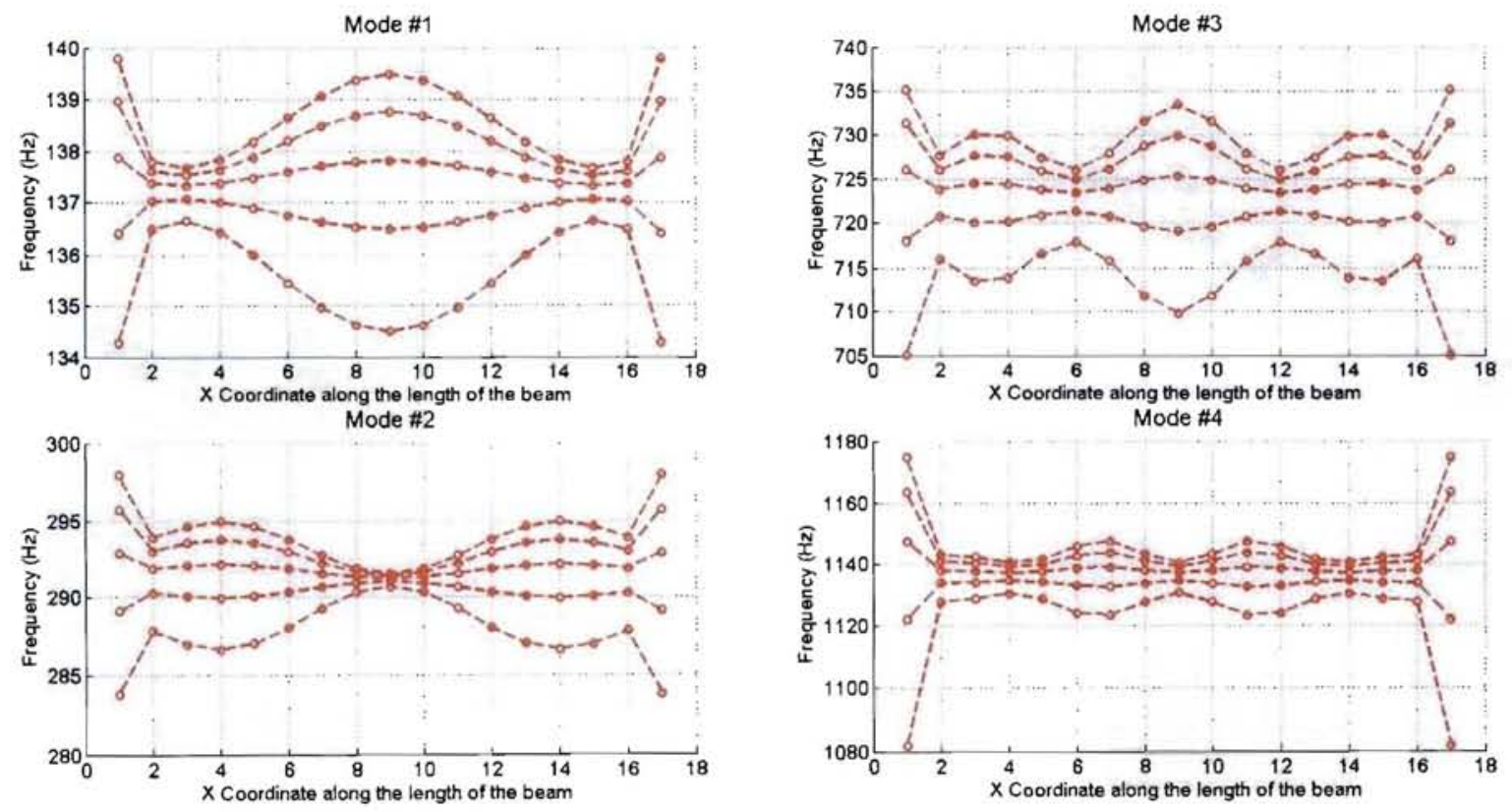

Figure 7: The influence of the defect location on the first four natural frequencies. 
A total of 85 computer runs are obtained each time varying the location of defect and the reduction factor for stiffness. The natural frequencies of the first four bending modes are observed to vary as demonstrated in Figure 7.

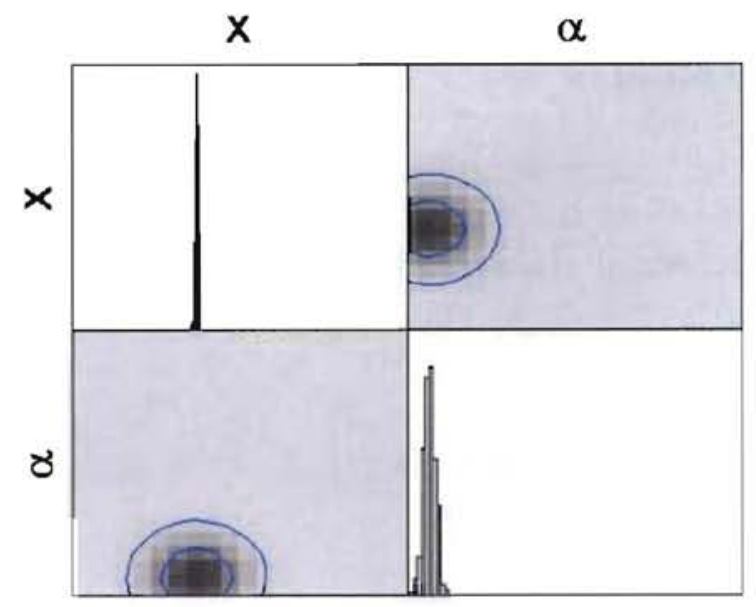

Figure 8: The posterior distributions of the two calibration parameters: location and severity of rock pocket defect.

A GPM is trained using the first four natural frequencies of the beam. Because only the natural frequencies are adapted herein, we cannot expect to locate the defect in a symmetric structure without incorporating spatial information, such as mode shapes. Clearly, two possible symmetric locations of defect will be equally plausible.

Then, according the procedure described in Section, the two parameters, which describe the location and extent of damage, are characterized. The posterior distributions of these two parameters after calibration are given in Figure 8.

Table The calibrated values of the parameters that define the location and severity of rock pocket defect.

\begin{tabular}{llll} 
Calibration Parameter & True Value & Calibrated Value (mean) \\
\hline Location of Defect $(\mathbf{x})$ & 7.0 units & 6.92 units \\
\hline Extent of Defect $(\boldsymbol{\alpha})$ & 0.750 & 0.753 \\
\hline
\end{tabular}

As seen in Table, the calibration approach successfully identified the location and extent of the rock-pocket defect - once the type of defect and the number of defects are known. It must be emphasized that these results present the best case scenario. However, in practical applications, we cannot expect this knowledge to be a priori available. 
Since in practical applications, the true number of defects will not be known, it is necessary to assume to define one variable for the location of the defect. Therefore, a successful defect detection technique should yield two distinct poles in the posterior distribution of the parameter $\mathrm{x}$. However, when the same approach is applied to the case illustrated in Figure 9, instead of distinguishing between these two closely spaced defects, the calibration yields a defect location somewhere between the two actual defects. It predicts one single defect with a higher level of stiffness reduction- in other words; it smears the effects of two defects into a single, but larger one. This is an example of a very general problem where the influence of a defect at one location on the measured quantities is identical to the influence of a defect of different severity or even type located at some other location in the system. These results, not shown here in the interest of brevity, raise the problem of uniqueness and emphasize the importance of the quality and quantity of experimental information.

- Scenario \#2: Detecting two Rock-pocket defects using natural frequencies and mode shapes.

Perhaps the most important problem during calibration based damage or defect detection is the uniqueness: several different combinations of calibration parameters can make the FE model match the experiments. Such a problem is observed in the previous scenario while attempting to detect more than one defect using only the natural frequencies. One obvious remedy to the problem is increasing the quantity of experimental information. In this scenario, the FE model will be calibrated using both natural frequency and mode shape information to detect two rock pocket defects as illustrated in Figure 9.

reduction factor for stiffness, $\alpha: 0.75$

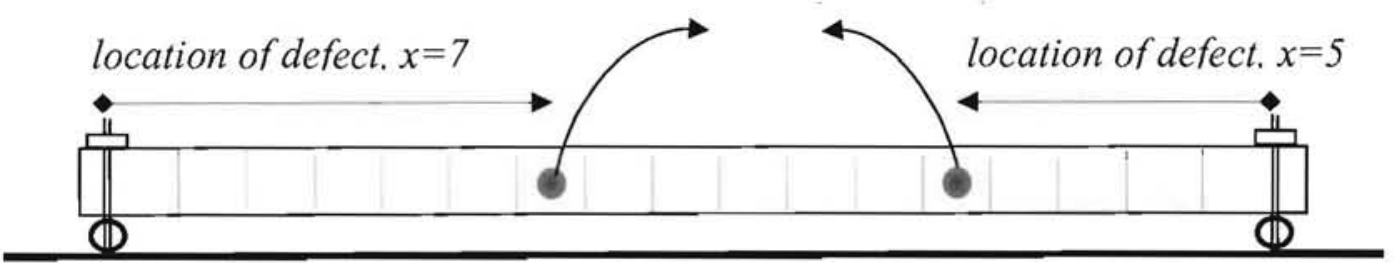

Figure 9: Characteristics of the two virtual defects simulated using the finite element model.

The reinforced concrete beam FE model with two virtual rock pocket defects, as illustrated in Figure 9, is used to generate simulated experimental data. The defects are located at the seventh and thirteenth compartments. Consistent with the previous scenario, the first four bending modes are used as defect indicators. The mode shapes were defined for the vertical deflections of the 18 equidistant points at the center of the beam width. Mode shape vectors are high dimensional and make the statistical inference computationally expensive. Therefore, singular value decomposition is applied to reduce the dimensionality of mode shape vectors. A total of four singular values are used to represent the mode-shape vectors. 
The FE model is calibrated to retrieve the 'correct' values for $\mathrm{x}$ and $\alpha$ parameters used to define the defects. The posterior distributions, given in Figure 10, illustrate two poles for the location of defect- proving the possibility of detecting more than one defect even though the true number of defects is unknown.

The difference in the posterior distributions of the two scenarios, with one defect and two defects, emphasizes the challenge in detecting multiple defects when the true number of defects is unknown. However, although the levels of uncertainty in the posterior distributions are significantly increased - meaning that the influence of defects are spread over a larger area- the proposed method still delivers viable information regarding the location and severity of defects. In cases where more than one defect is present, the outcome of most concern is the ability to determine the number and presence of defects. The results presented in Figure 10 can be considered satisfactory for such purposes. Perhaps one can repeat the calibration procedure defining two distinct parameters ( $\mathrm{x} 1$ and $\mathrm{x} 2)$ as well as two distinct reduction factors ( $\alpha 1$ and $\alpha 2)$ to better identify the location as well as the severity of the defect.

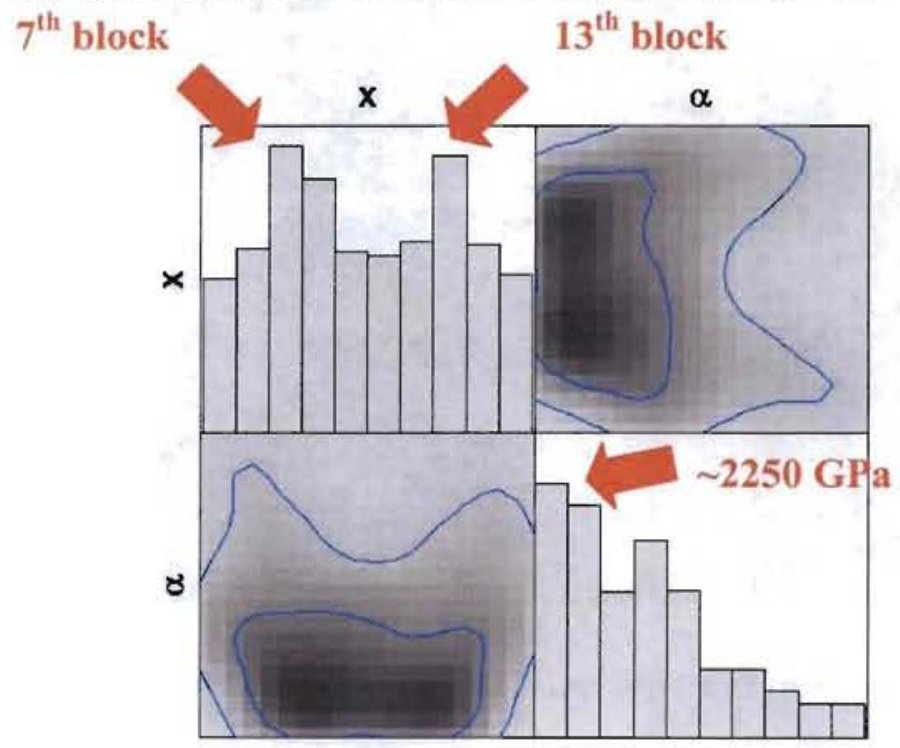

Figure 10: The posterior distributions of the two calibration parameters: location and severity of defect.

As a result, using simulated data, we can successfully detect, locate and quantify defects in concrete members. Clearly, it is easier to identify defects using simulated experimental information. The effectiveness of inverse methods must, however, be tested using real physics measurements, because methods which perform well with simulated data may struggle when dealing with measurement errors and inadequate simulation models. To illustrate the potential of the method in practical application, laboratory scaled beam models are built and tested. When dealing with real measurements the following problems are anticipated to raise: (a) the defect present in the test beams is unavoidably different than the defect model represented in the FE model; (b) the boundary conditions of the beam during testing is, again unavoidably, different 
then the idealized boundary conditions in the FE model. The findings of this study are presented in the following sections.

\section{Test Beams:}

Test beam is a three lightly reinforced concrete beam, $15 \mathrm{~cm}$ by $22.5 \mathrm{~cm}$ in cross section and $1.95 \mathrm{~m}$ in length. The beam is supported with roller suppers at both ends. To prevent rattling of the beam, the beam is clamped through two 5/8" steel between the roller supports provided both at the bottom and top surface of the beam.
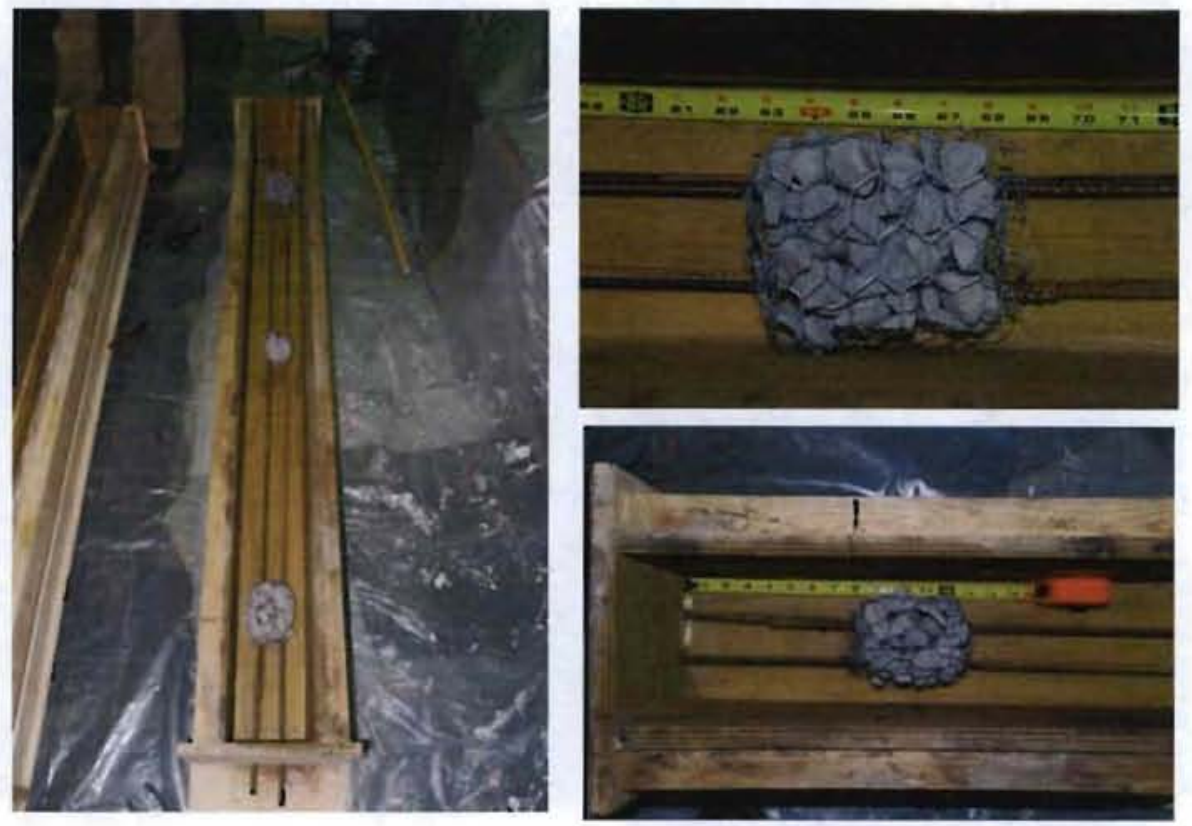

Figure 11: Rock pocket defects are intentionally located in the concrete beam.

. The rock pocket defects are created with aggregates placed in a net before the concrete was poured, see Figure 11. Approximate locations of these rock pockets are provided in Figure 12.

\section{Location of defects}

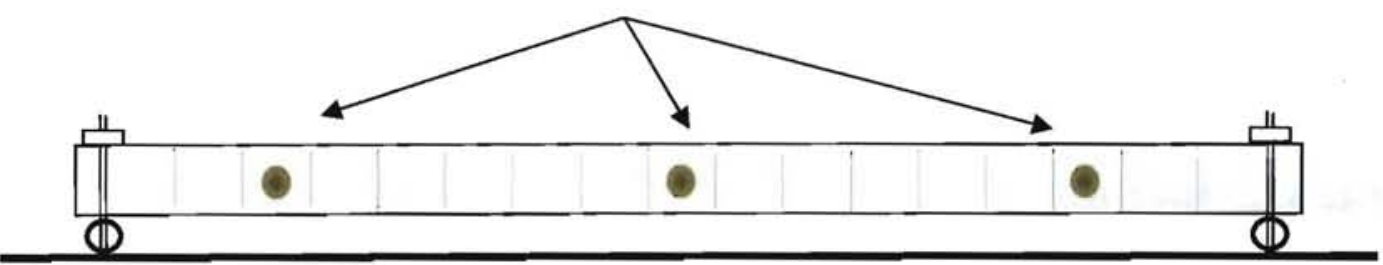

Figure 12: The location of rock pocket defects. 


\section{Experiments:}

The experiments are conducted to obtain the time history response of the beams to a hammer impact excitation. Accelerations at 16 points located equidistant are measured using accelerometers. Vibration measurements are strictly limited to the vertical vibration of the beam to be relevant to practical application where only the top of the beam would be accessible for testing. Two locations, one approximately at quarter-length (excitation \#1 in Figure 13) and one at approximately at mid-length (excitation $\# 2$ in Figure 13) of the beam, are excited with an impact hammer, see Figure 14.

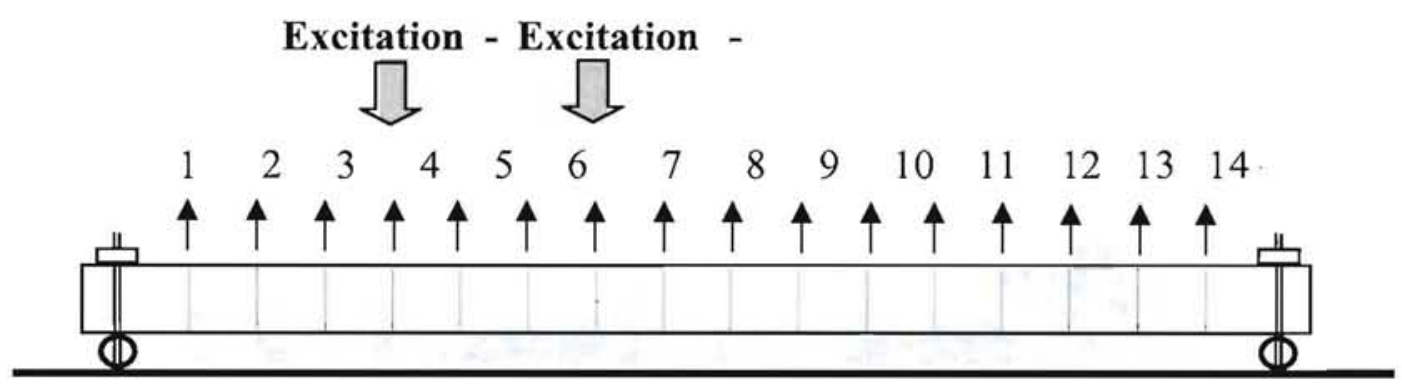

Figure 13: Experimental set-up includes 16 measurement location and two excitation locations.
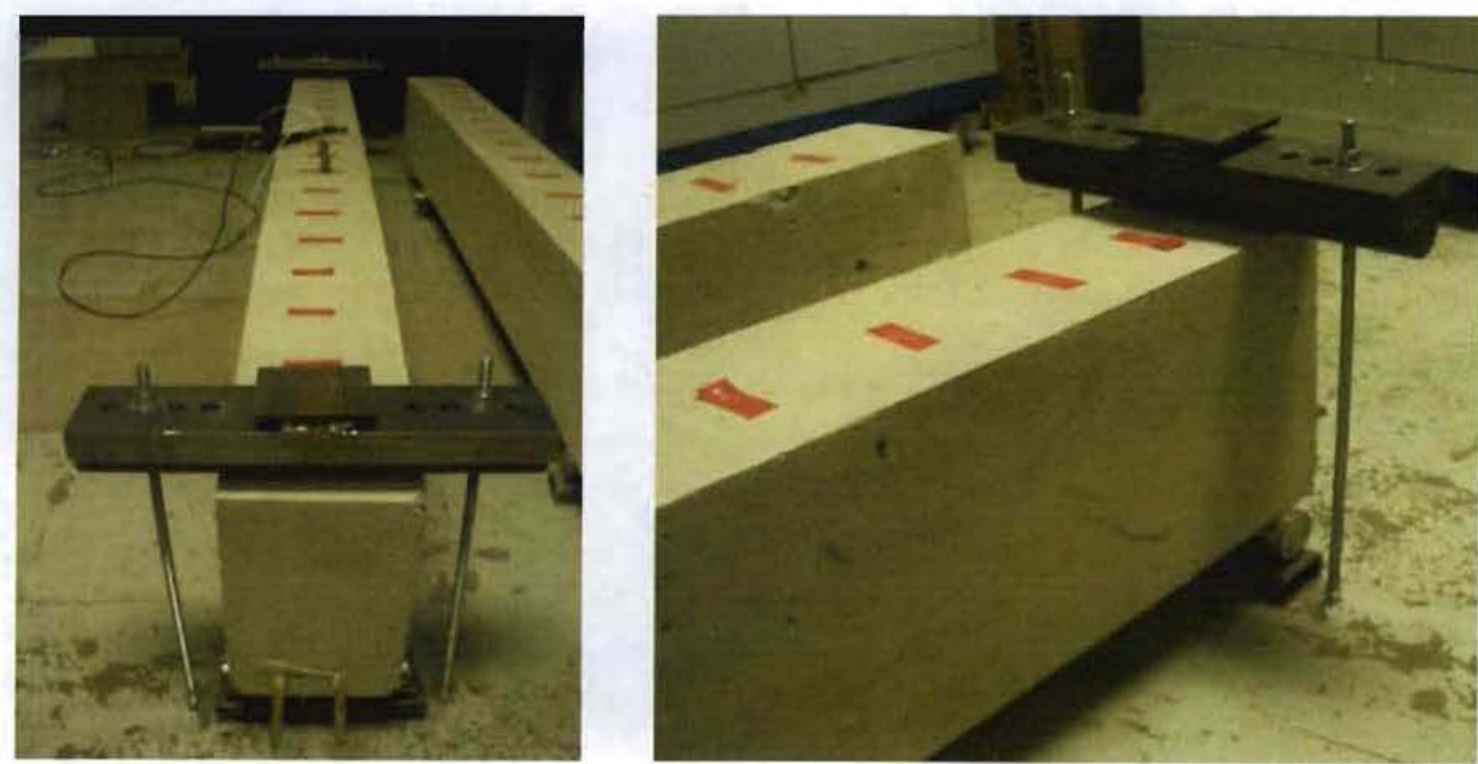

Figure 14: The boundary conditions of the beams are designed to provide simple support and at the same time to prevent the rocking behavior of the beam. 


\section{Feature Extraction:}

The FRFs, obtained exciting point \#7, are illustrated in Figure 15. A number of modes are observed between $40 \mathrm{~Hz}$ and $450 \mathrm{~Hz}$. Because of the complexity of the boundary conditions, some of these modes are noted to be a result of the coupling of beam modes and the supporting system.

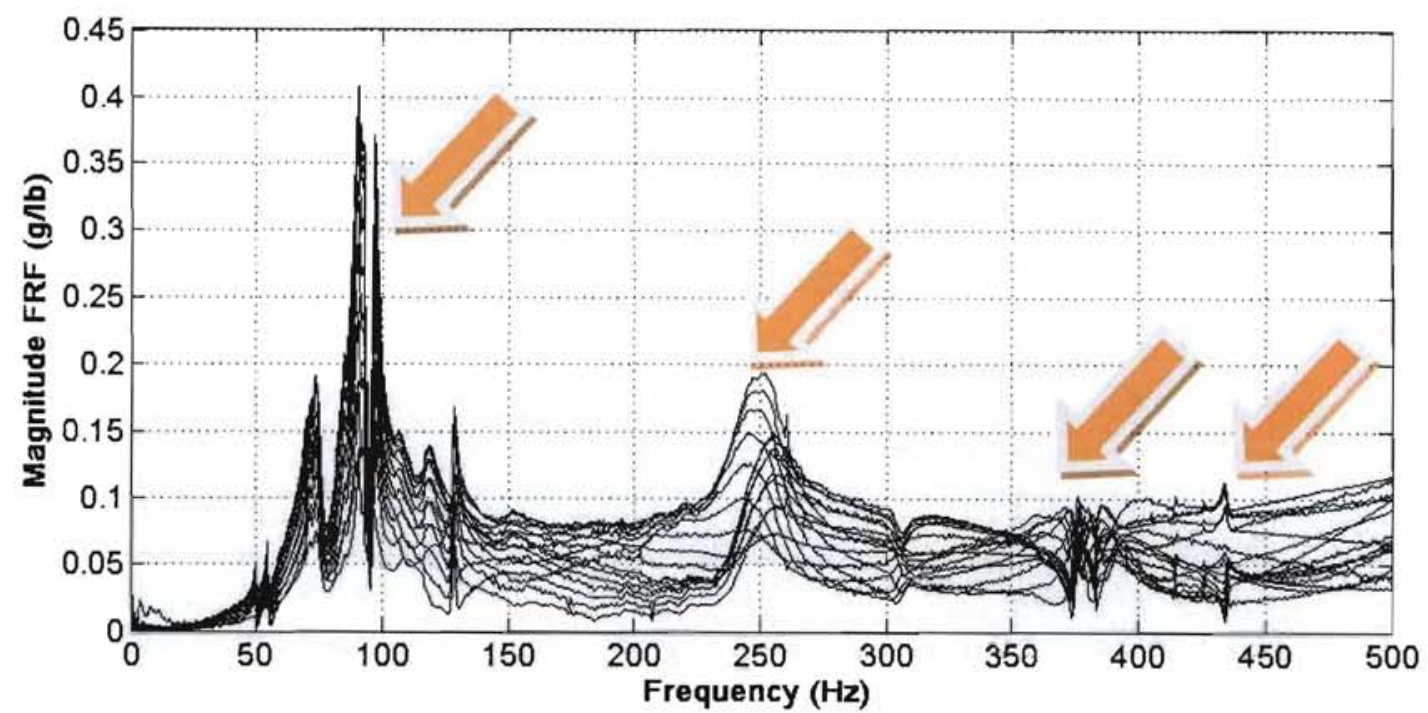

Figure 15: The FRF of beam with rock pockets identified frequencies are illustrated with read dashed lines: (top) excitation 1, (bottom) excitation 2.

Feature extraction is completed using rational fraction polynomials method (Richardson and Formenti, 1982). A total of four bending modes are observed within tested frequency range. The frequencies of these modes are given in Table, while the mode shapes are presented in Figure 16.

Table The natural frequencies obtained from the beam with rock pockets.

\begin{tabular}{l:l} 
Mode & Frequency \\
\hline 1 & $89.2 \mathrm{~Hz}$ \\
\hline 2 & $242 \mathrm{~Hz}$ \\
\hline 3 & $380 \mathrm{~Hz}$ \\
\hline 4 & $435 \mathrm{~Hz}$
\end{tabular}

The variability of physical experiments can only be studied by repeated experiments. However, the experiments are typically costly and time-consuming relative to the FE models. It is a common application to repeat only a portion of the experiment. For instance, in a test setup with $\mathrm{k}$ measurement points, repeating the experiments at a select few measurement points $(<<\mathrm{k}) \mathrm{can}$ reduce the required resources yet can still yield reasonable information about the inherent 
variability in the experiment. The driving point measurements at point \#7 are repeated 16 times to obtain information about the experimental uncertainty.
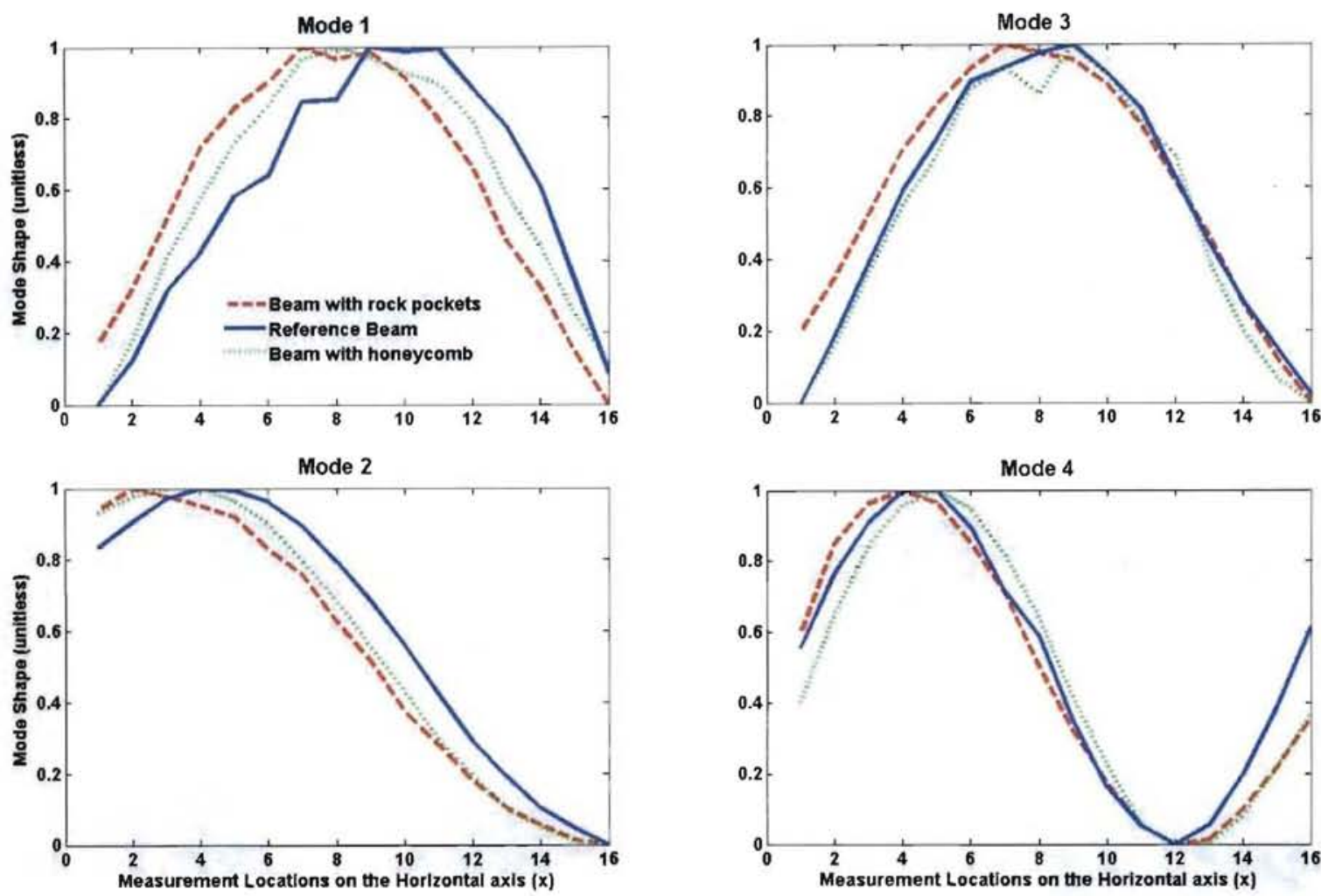

Figure 16: The first four mode shapes of the beams.

\section{Numerical Models:}

Defect detection of a simply supported beam is a simple enough problem that can be tackled using closed form solutions developed for continuous systems, however a discrete model approach add versatility in the application of the procedure to systems of various geometry and complexity. Discretization of the model does not influence the nature of the defect detection problem. The discrete numerical models are built in ANSYS v. 11 using solid 95 elements. The beams are built based on linearly elastic behavior assumption. This assumption is reasonable as the vibration levels induced during the tests are low. Moreover, the defects are not anticipated to induce nonlinearity.

The boundary conditions are represented with two springs exerting vertical restraints both at the top and bottom surface of the beam [Figure 17]. The spring constants are assumed to be symmetric for both ends - which is with all likelihood is not the case, because variation in assembly is a routinely experienced practical problem. 
K1: Linear spring

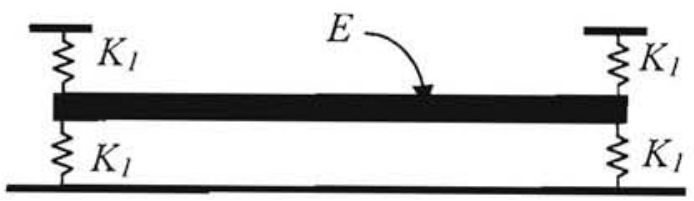

K2: Linear spring

Figure 17: The configuration of the finite element model boundary conditions.

The FE model is build to have 17 sections each of which can have independent young's modulus and density. The corresponding mode shapes obtained by the beam FE model are illustrated in Figure 18.
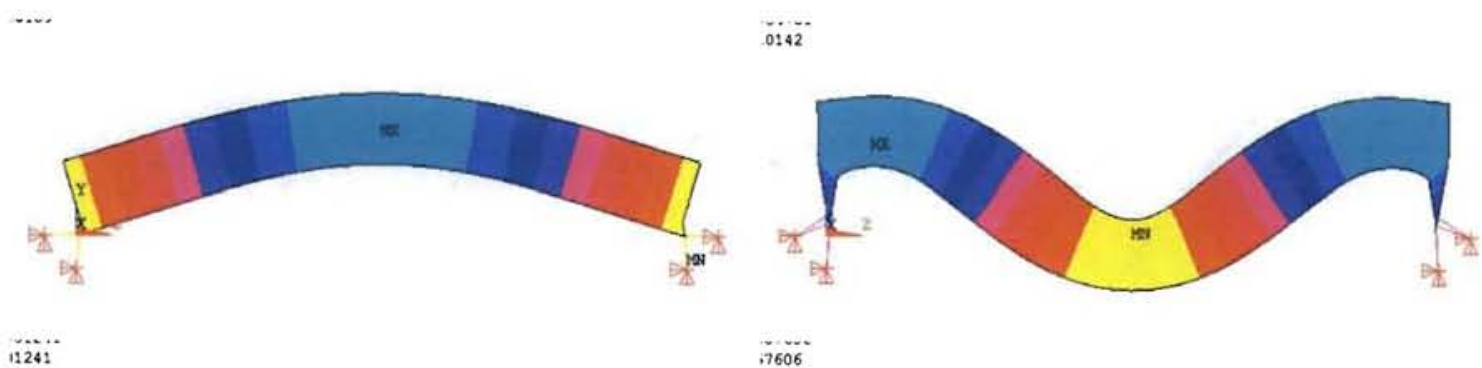

1241

inosis
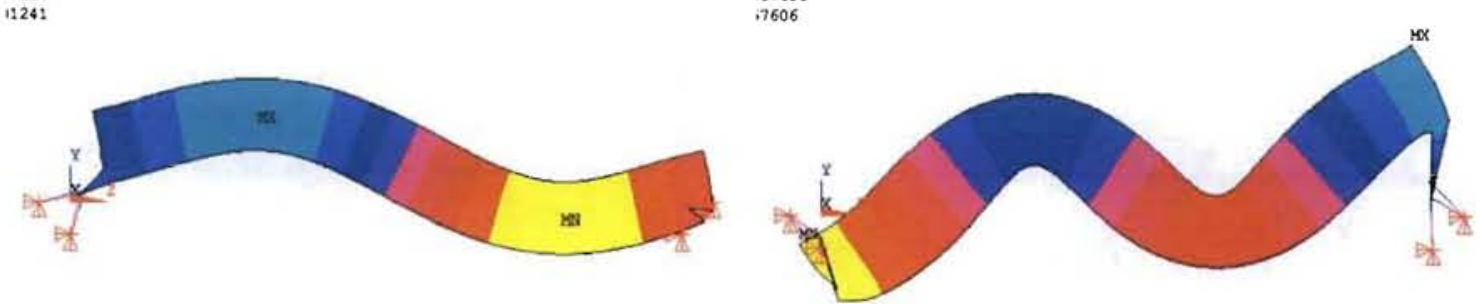

Figure 18: The first four mode shapes predicted by the finite element model.

\section{Determination of Defects in Test Beam:}

The difficulty of this particular problem is due to complexity of the boundary conditions of the test beams [Figure 14]. The precise values of the spring constants, which define the boundary conditions, are unknown. Typically, beam vibrations are measured under free-free boundary conditions to alleviate the problems due to difficulty of representing fixed boundary conditions in laboratory conditions. However to have any practical value, a defect detection approach must also be applicable under conditions commonly found in, for instance, prestressed precast reinforced concrete beam manufacturing.

The spring constants representing the boundary conditions are approximated by calculating the axial stiffness of the steel rods used to clamp the beam at both ends. Although, this calculated value is expected to be imprecise, it is not defined as a calibration parameter. The significant differences between the initial FE model predictions and the experimental measurements are shown in Table. 


\begin{tabular}{ll|l} 
Mode & Experimental & FE Model (mean) \\
& Frequency & Before Calibration \\
\hline 1 & $89.2 \mathrm{~Hz}$ & $95 \mathrm{~Hz}$ \\
\hline 2 & $242 \mathrm{~Hz}$ & $255 \mathrm{~Hz}$ \\
\hline 3 & $380 \mathrm{~Hz}$ & $390 \mathrm{~Hz}$ \\
\hline
\end{tabular}

The $\mathrm{x}$ and $\alpha$ parameters are calculated using various levels of experimental information: only the natural frequencies (see scenario 1 in section 4), natural frequencies along with the singular values of the mode shapes (see scenario 1 in section 4 ) and using all three mode shape vectors as well as natural frequencies. In either of these three cases, the correct location of the defects is not obtained.

The posterior distributions obtained using the first three natural frequencies and mode shape vectors are illustrated in Figure 19. Although the severity of the defect seems to be identified relatively accurately, the effects of three defects are lumped into one single location. The reasonable success obtained when two defects were simulated in Section 4 is not present in this particular case. The explanation may be because (1) defects in numbers larger than two are significantly difficult to distinguish, (2) the imprecision of physical experiments degrade the success of the inference process, (3) the inaccuracies in the numerical model through the boundary conditions is interfering with the inference process.

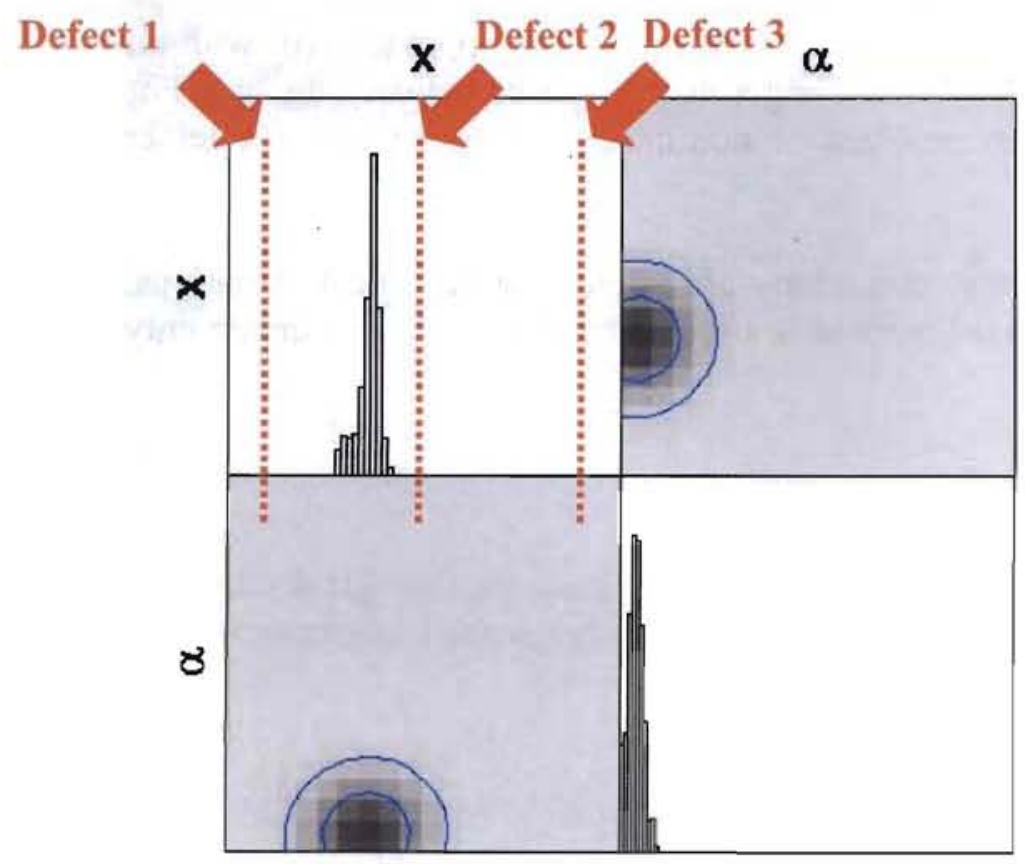

Figure 19:The posterior distributions obtained for the test beam defect locations. 


\section{Discussion and Conclusion:}

In this paper, a general procedure for defect detection in concrete construction is illustrated. A method, which can adjust the boundary conditions and simultaneously detect the location, extent and type of damage, is of great value.

The proposed approach does not depend upon the type of the defect, the structure of interest or the measured quantities. The proposed approach relies on the fact that structural with and without defects would yield different dynamic signatures. By comparing the dynamic response of a concrete member with and without defects, one can, theoretically, determine the presence of defects. Therefore, we conclude that FE modeling coupled with nondestructive testing can provide a viable solution to detection of internal defects in concrete members.

Although the actual implementation of the proposed methodology will be application specific, its generality allows it the use of various forms of experimental information. Moreover, the use of discrete method add further versatility such that the method can be deployed to structural forms of varying geometry and complexity.

However, if a defect is smaller than the defect size which would result in detectable and measurable change in the dynamic response, its influence will be smeared in the environmental effects, such as ambient vibration, measurement noise...etc. Therefore, a defect detection approach based on vibration measurements, as in every nondestructive technique, has a lower defect size limit for which it can be deployed. Also, for two closely spaced cracks, unless a large amount of accurate experimental information is available, the calibration approach will with all likelihood smear the effects of these two defects into a larger defect located between the two. For defects which are spaced farther apart, the problem of non-unique solutions poses a lesser problem.

The systems of interest and their operational conditions are subject to statistical variations, therefore in practical applications, the proposed method is anticipated to yield higher uncertainty compared to those presented herein.

\section{Acknowledgements:}

Elizabetta Pistone's help during vibration experiments is appreciated. Author thanks to Paul Kremer and the work-study students of Pennsylvania State University for their assistance during the casting of the concrete beam specimens.

\section{References:}

Aoki, T., Sabia, D., Rivella, D., Muto, H., (2005), "Dynamic Identification and Model Updating of the Howa Brick Chimney, Tokoname, Japan," Structural Studies, Repairs and Maintenance of Heritage Architecture, IX, Vol. 83, pp. 265- 275. 
Doebling, S., Farrar, C. R., Prime, M. B. (1998), 'A summary review of vibration-based damage identification methods," Shock and Vibration Digest, Vol. 30, No. 91, pp. 91- 105.

Higdon, D., Gattiker, J., Williams, B., Rightley, M., (2008), "Computer Model Calibration Using High-dimensional Output," Journal of the American Statistical Association, Vol. 103, No. 482, pp. 570-583.

Hogg, R. V., and Craig, A. T., (1978), "Introduction to Mathematical Statistics," Macmillan.

Kennedy, M., O'Hagan, A., (2000), "Predicting the Output from a Complex Computer Code When Fast Approximations are Available," Biometrika, Vol. 87, pp. 1-13.

Richardson M. H., Formenti, D. L., (1982), "Parameter Estimation From Frequency Response Measurements Using Rational Fraction Polynomials," Proceedings of the $1^{\text {st }}$ International Modal Analysis Conference, Orlando, FL.

Sohn, H., Farrar, C. R., Hemez, M. F., Shunk, D. D., Stinemates, D. W., Nadler, B. R., Czarnecki, J. J., (2004), "A review of Structural Health Monitoring Literature: 1996- 2001" Los Alamos National Laboratory Report, LA-13976-MS.

Vestroni, F., (2008), “ Structural Identification and Damage Detection," Dynamic Methods for Damage Detection in Structures, Springer-Verlag, Austria. 
